\title{
Sistema cobit en los procesos de auditorías de los sistemas informáticos
}

\section{System cobit processes audits of computer systems}

\author{
Julio Jhovany Santacruz Espinoza ${ }^{1, *}$, Cesar Remigio Vega Abad ${ }^{1, \dagger}$, \\ Luis Fernando Pinos Castillo ${ }^{1, \ddagger}$, y Oscar Efrén Cárdenas Villavicencio ${ }^{2, \otimes}$. \\ ${ }^{1}$ Universidad Católica de Cuenca, Ecuador. \\ ${ }^{2}$ Universidad Técnica de Machala, Ecuador. \\ \{jsantacruze,crvegaa,Ifpinosc\}@ucacue.edu.ec, oecardenas@utmachala.edu.ec
}

Fecha de recepción: 08 de Noviembre de 2017 — Fecha de aceptación: 14 de Diciembre de 2017

DOI: https://doi.org/10.26910/issn.2528-8083vol2iss8.2017pp65-68

\begin{abstract}
Resumen-EL presente trabajo de investigación ha sido realizado para mostrar los beneficios del uso de sistema COBIT en los procesos de auditorías de los sistemas informáticos, la problemática se relaciona en ¿Cómo incide en el proceso de auditorías en las instituciones, empleo del sistema COBIT?, el objetivo principal es identificar la incidencia del uso del sistema COBIT en el proceso de auditorías empleados por os sistemas informáticos dentro de las organizaciones tanto de carácter público como privado; con el fin de lograr nuestros objetivos planteados de la investigación se desarrollará primeramente con la conceptualización de términos claves para un fácil entendimiento de la temática, como conclusión: podemos decir el sistema COBIT permite identificar la metodología mediante la utilización de información de los departamentos informáticos, para determinar los recursos de la (T.I) Tecnología de la información, especificados en el sistema COBIT, como son los archivos, programas, redes informáticas incluidos el personal que los utiliza o manipula la información, con el propósito de proveer la información que la organización o empresa requiere para conseguir sus objetivos.
\end{abstract}

Palabras Clave-COBIT, Tecnologías de la Información, Auditoria, Sistemas Informáticos.

Abstract-The present research work has been carried out to show the benefits of the use of the COBIT system in the auditing processes of the computer systems, the problem is related to: How does it affect the process of audits in the institutions, use of the COBIT system? The main objective is to identify the incidence of the use of the COBIT system in the auditing process used by computer systems within both public and private organizations; In order to achieve our stated objectives of the research will be developed first with the conceptualization of key terms for an easy understanding of the subject, as a conclusion: we can say the COBIT system allows to identify the methodology by using information from the IT departments, to determine the resources of the (IT) Information Technology, specified in the COBIT system, such as files, programs, computer networks, including personnel that use or manipulate the information, with the purpose of providing information that the organization or company requires to achieve its objectives.

Keywords-COBIT, Information Technologies, Auditing, Computer Systems.

\section{INTRODUCCIÓN}

$\mathbf{E}$ 1 presente trabajo de investigación se refiere al uso del sistema COBIT que se puede definir como una guía o modelo para realizar auditorías de la gestión y control de los sistemas de información y tecnología, orientado a los departamentos informáticos de una organización, es decir a los auditores involucrados en el proceso.

Las principales características del Sistema COBIT, están orientadas al negocio u organización, pueden ser utilizadas por los usuarios y a su vez por los auditores, como una lista de verificación minuciosa para los encargados de cada proceso, este sistema está formado con estándares de control y auditoria (COSO), basado en una observación crítica y analítica de las actividades en las TI.

La investigación se realizó con el propósito de relevar la

\footnotetext{
*Magister en Redes de Comunicaciones

${ }^{\dagger}$ Magister en Redes de Comunicaciones

$\ddagger$ Magister en Redes de Comunicaciones

${ }^{\otimes}$ Magister en Telecomunicaciones
}

importancia para comprender que, a pesar de ser un procedimiento que puede considerarse a ser de un costo alto en tiempo y dinero, su utilización de forma adecuada puede generar beneficios operativos, administrativos y económicos en un corto, mediano y largo plazo, en cualquier organización, empresa o negocio, depende de cada una de las características de las instituciones antes mencionadas, es oportuno y necesario realizar la adaptación del presente sistema y usar lo necesario para un correcto funcionamiento.

\section{DESARROLLO}

Ante la necesidad de crear y fortalecer el ambiente de control de las tecnología de la información que soporta las operaciones de las empresas y su esquema de gobierno, se hace oportuna la definición de un marco de referencia. 


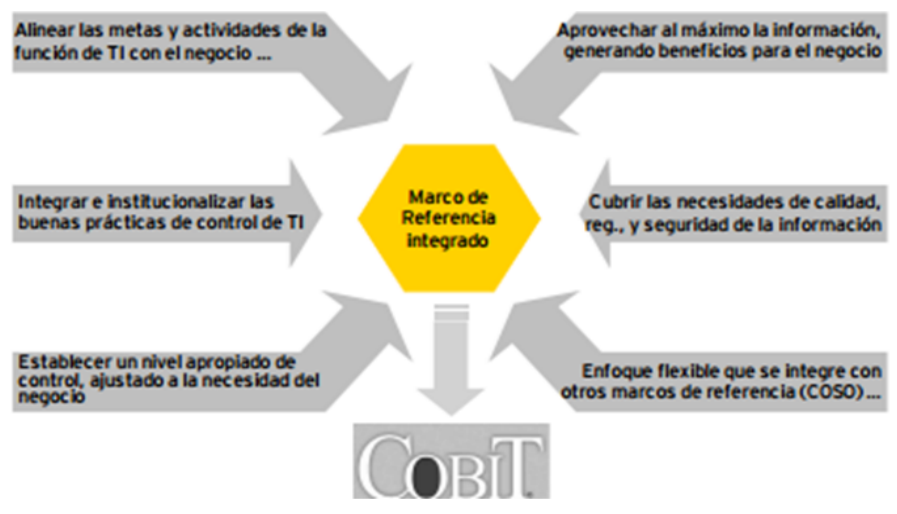

Figura 1. Marco de referencia integrado de COBIT

Fuente: (Javeriana, 2012)

Según Zambrano (2014),'El Instituto de Gobierno de TI fue formado por la Auditoría de Sistemas de Información y de la Asociación de Control (ISACA) y su Fundación asociada en 1998 para avanzar en el entendimiento y la adopción de principios de gobierno de TI".Con la unión de las Directrices Gerenciales en la tercera edición de COBIT, su expansión y mayor cubrimiento sobre el Gobierno de TI, el Instituto de Gobierno de TI adquirió un la participación de liderazgo en el desarrollo de la publicación. EL Sistema COBIT cuando se inició estaba basado en los Objetivos de Control de la ISACF, a medida que paso el tiempos se realizaron mejoras al sistema, es por esta razón que en la actualizada el sistema cuenta con los estándares internacionales a nivel técnico, profesional, especifico y regulatorio dentro de la industria. Los Objetivos de Control resultantes han sido realizados para su utilización en los diferentes sistemas de información que maneje la empresa. El sistema COBIT ha desarrollado varias ediciones, la primera edición se publicó en el año 1996, la segunda edición se publicó después de dos años de haber publicado la primer edición eso se realizó en 1998, la tercera edición en 2000 (la edición disponible en internet estuvo disponible en 2003), y la cuarta edición en Diciembre de 2005, la versión 4.1 se publicó en está disponible desde mayo de 2007, la versión online de COBIT 5 ya está disponible.

\section{¿Qué es el sistema Cobit?}

"Las siglas COBIT significan Objetivos de Control para Tecnología de Información y Tecnologías relacionadas. El modelo es el resultado de una investigación con expertos de varios países, desarrollado por ISACA-Information Systems Audit and Control Association" (Reyes, 2015a).

El sistema COBIT, es el producto de un proyecto de varias personas de diferentes nacionalidades; con el interés de poder desarrollar un sistema que permita a la empresa poder obtener resultados de los procesos que se realizan dentro de cada empresa.

Según Almanza Gomez (2012), ÇOBIT es la para identificar Objetivos de Control para las Tecnologías de Información y Relacionadas. Este sistema de control está constituido por un conjunto de prácticas orientadas para el manejo de los procesos relativos a la información".

El Sistema de Control para las tecnologías de información y Relacionadas (COBIT), es construido por un conjunto de prácticas dirigidas al desarrollo de los procesos de la información, dentro de las que se destacan la distribución, almacenamiento y administración, permiten asegurar que los resultados, estén enfocados hacia los requerimientos del negocio empresa u organización.

COBIT, es una herramienta de gobierno de las tecnología de la información que ha cambiado la forma en que trabajan los profesionales de tecnología. Relacionando tecnología informática y prácticas de control, este sistema o modelo refuerza los estándares globales que sobresalen en un recurso crítico para la administración de gerencia, los profesionales de control y los auditores.

Este sistema se aplica a los sistemas de información de las diferentes empresas, donde se incluyen los pc o computadoras personales y las redes, está basado en la filosofía que los recursos de tecnología de información, necesitan ser administrados por un conjunto de procesos naturalmente agrupados para proveer la información pertinente y confiable que requiere una organización para lograr sus objetivos.

Según Reyes (2015b). "El sistema COBIT es precisamente un modelo para auditar la gestión y control de los sistemas de información y tecnología, orientado a todos los sectores de una organización, es decir, administradores, usuarios y por supuesto, los auditores involucrados en el proceso".

EL COBIT es un sistema o modelo de evaluación y monitoreo que enfatiza en el control de negocios y la seguridad y que abarca controles específicos, desde una visión de negocios.

\section{¿Cuál es el propósito de COBIT 5?}

Uno de los propósitos por lo cual se desarrolló el sistema COBIT, fue para facilitar y ayudar a las organizaciones a obtener el valor óptimo de la tecnología de la información conservando el balance entre la realización de beneficios, la utilización de recursos y los fases de riesgo asumidos.

COBIT 5 posibilita que la tecnología de la información sea manejada y gestionada de forma conceptual para toda la organización, teniendo en cuenta el negocio, o empresa y sus principales y funcionales de punta a punta así como los interesados internos y externos.

Este sistema se puede utilizar y aplicar en organizaciones de diferentes tipos y todos los tamaños, sin importar el sector de desarrollo de la empresa puede ser privado, público o entidades sin fines de lucro.

\section{¿Quién utiliza COBIT?}

El sistema COBIT es empleado en todo el mundo por personas quienes tienen como responsabilidad principal los procesos de negocio y la tecnología, son aquellos de quien depende la tecnología y la información confiable, fiable y los que proveen calidad, confiabilidad y control de tecnología de información.

Este sistema se basa 5 principios y 7 habilitadores, COBIT 5 utiliza prácticas de gobierno y gestión para describir las acciones que son ejemplo de mejores prácticas de su aplicación.

\section{Principios de COBIT}


1. Satisfacer las necesidades del accionista.

2. Considerar la empresa de punta a punta.

3. Aplicar un único modelo de referencia integrado.

4. Posibilitar un enfoque holístico.

5. Separar gobierno de la gestión.

\section{Habilitadores de COBIT}

1. Principios, políticas y modelos de referencia.

2. Procesos.

3. Estructuras organizacionales.

4. Cultura, ética y comportamiento.

5. Información.

6. Servicios, infraestructura y aplicaciones.

7. Gente, habilidades y competencias.

El gobierno asegura que los objetivos empresariales se logran evaluando las necesidades de los accionistas, las condiciones y opciones; establecer la dirección a través de la priorización y la toma de decisiones; y monitorear el desempeño, el cumplimiento y el progreso versus la dirección y objetivos acordados (EDM, por Evaluar, Dirigir, Monitorear).

Por su parte la gestión se ocupa de planificar, construir, ejecutar y monitorear las actividades alineadas con la dirección establecida por el organismo de gobierno para el logro de los objetivos empresariales (PBRM ó Planificar, Construir, Ejecutar y Monitorear, por su sigla en inglés) (Sperat, 2016).

\section{Procesos indentificados por la COBIT}

La COBIT define las actividades TO en un modelo general de procesos compuesto por cuatro dominios: Planificar y Organizar (PO), Adquirir e Implementar (AI), Proveer y Soportar (DS) y Monitorear y Evaluar (ME). Esto dominios se corresponden con las áreas de responsabilidad tradicionales: planificar, construir, ejecutar y monitorear (Saffirio C., 2007).

\section{COBIT: Un Marco de referencia para la Información y la Tecnología}

Las empresas poseen un capital activo muy valioso: información y tecnología. Cada vez en mayor medida, el éxito de una empresa depende de la comprensión de ambos componentes. Las buenas prácticas concentradas en el marco de referencia COBIT, permiten que los negocios se alineen con la tecnología de la información para así alcanzar los mejores resultados.

La información y la tecnología que la soporta simbolizan los activos más valiosos de muchas empresas, aunque con frecuencia son poco entendidos. Las empresas exitosas reconocen los beneficios de la tecnología de información y la utilizan para impulsar el valor de sus interesados (stakeholders). Estas empresas también entienden y administran los riesgos asociados, es decir, el aumento en los requerimientos regulatorios, así como también una gran dependencia de muchos de los procesos de negocio en TI. Pero todos estos elementos son clave para el gobierno de la empresa. El valor, el riesgo y el control constituyen la esencia del gobierno de TI.

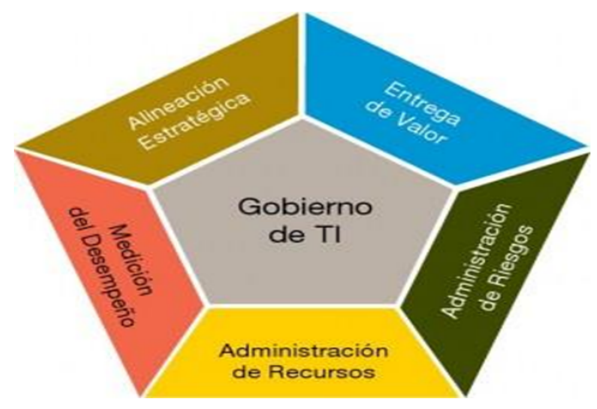

Figura 2. Gobierno de TI

Fuente: (BITCompany, 2015)

La orientación al negocio que realiza COBIT consiste en vincular las metas del negocio con las metas de TI, brindando métricas y modelos de madurez para medir los logros, e identificando las responsabilidades asociadas de los propietarios de los procesos de negocio y de TI. El enfoque hacia procesos de COBIT se ilustra con un modelo de procesos, el cual subdivide TI en 34 procesos de acuerdo a las responsabilidades de planear (planes de auditoria), construir (Implementar los distintos planes de auditoria), ejecutar (Ejecutar las diferentes planeaciones) y monitorear (verificar y controlar los procesos de ejecución); de esta manera, se ofrece una visión de punta a punta de la TI. El concepto de arquitectura empresarial ayuda a identificar aquellos recursos esenciales para el éxito de los procesos, es decir, aplicaciones, información, infraestructura y personas. En resumen, para proporcionar la información que la empresa necesita de acuerdo a sus objetivos, los recursos de TI deben ser administrados por un conjunto de procesos agrupados de forma natural que serán controlados a través de la auditoria al momento que se realice implementando el sistema COBIT.

Una respuesta al requerimiento de determinar y monitorear el nivel apropiado de control y desempeño de TI, son los conceptos que COBIT define específicamente:

- Benchmarking de la capacidad de los procesos de TI Son modelos de madurez derivados del Modelo de Madurez de la Capacidad del Instituto de Ingeniería de Software

- Metas y métricas de los procesos de TI para definir y medir sus resultados y su desempeño, basados en los principios de balanced business Scorecard de Robert Kaplan y David Norton. Es decir, este proceso es para controlar y optimizar el desempeño de las empresas.

- Objetivos de las actividades para controlar estos procesos, con base en los objetivos de control detallados de COBIT.

La evaluación de la capacidad de los procesos basada en los modelos de madurez de COBIT es una parte clave de la implementación del gobierno de TI. Después de identificar los procesos y controles críticos de TI, el modelado de la madurez permite identificar y demostrar a la dirección las brechas en la capacidad de mejorar y optimizar los recursos de TI para de esta manera concretar los objetivos en los que debe enfocarse la empresa y alcanzar buenos resultados. 
Tabla 1. Cuadro Comparativo del Sistema Cobit con otros Sistemas Como (Itil)

\begin{tabular}{|c|c|c|}
\hline & COBIT & ITIL \\
\hline Características & $\begin{array}{l}\text { Está,basado en la } \\
\text { filosofía de que } \\
\text { los recursos } \\
\text { de Ti necesitan } \\
\text { ser agrupados. } \\
\text { Diseñado como } \\
\text { un estándar } \\
\text { aceptado y ajustable } \\
\text { a las buenas } \\
\text { prácticas de seguridad } \\
\text { y control en TIC. } \\
\text { Es práctica y } \\
\text { simple. } \\
\text { Ayuda a la gerencia a } \\
\text { comprender y } \\
\text { asociar los riesgos } \\
\text { administrados,con } \\
\text { TIC. }\end{array}$ & $\begin{array}{l}\text { - Mejorar el servicio } \\
\text { al cliente. } \\
\text { - Propone el } \\
\text { establecimiento } \\
\text { de estándares. } \\
\text { - No es rígido en } \\
\text { cuanto a su } \\
\text { implementación. }\end{array}$ \\
\hline Ventajas & $\begin{array}{l}\text { - Mejora la calidad } \\
\text { y medición de las TI. } \\
\text { - Ayuda a implementar } \\
\text { un sistema de control. } \\
\text { - Presenta las } \\
\text { actividades en una } \\
\text { estructura manejable } \\
\text { y lógica. }\end{array}$ & $\begin{array}{l}\text { - TI desarrolla una } \\
\text { estructura más clara } \\
\text { y se centra en los } \\
\text { objetivos de la } \\
\text { organización. } \\
\text { - La administración } \\
\text { tiene un mayor control. } \\
\text { - Los cambios resultan } \\
\text { más fáciles de manejar. } \\
\text { - Mejor uso de los } \\
\text { recursos y reducción } \\
\text { de costos. }\end{array}$ \\
\hline Desventajas & $\begin{array}{l}\text { - Las buenas } \\
\text { prácticas de cobit } \\
\text { están enfocadas } \\
\text { en el control y no } \\
\text { en la ejecución. } \\
\text { - El marco de } \\
\text { referencia } \\
\text { mejora las áreas } \\
\text { TI desde el punto } \\
\text { de vista del } \\
\text { gobierno corporativo. }\end{array}$ & $\begin{array}{l}\text { - Tiempo y esfuerzo } \\
\text { necesario para su } \\
\text { implementación. } \\
\text { - La mejora del servicio } \\
\text { y reducción de costos } \\
\text { podría no ser visible. } \\
\text { - Su lenguaje y } \\
\text { terminología aún está } \\
\text { muy limitada a un } \\
\text { área muy pequeña de TI. } \\
\text { - La mayoría de } \\
\text { las organizaciones } \\
\text { complican demasiado } \\
\text { la implementación } \\
\text { de ITIL y esto lleva } \\
\text { en muchos casos } \\
\text { al fracaso. }\end{array}$ \\
\hline Ciclo de Vida & $\begin{array}{l}\text { - Dominios } \\
\text { - Procesos } \\
\text { - Actividades }\end{array}$ & $\begin{array}{l}\text { - Estrategia. } \\
\text { - Diseño. } \\
\text { - Transición. } \\
\text { - Operación. } \\
\text { - Mejora continua. }\end{array}$ \\
\hline Objetivos & $\begin{array}{l}\text { Lograr un balance } \\
\text { óptimo entre } \\
\text { las oportunidades } \\
\text { de tecnología de } \\
\text { información y } \\
\text { los requerimientos } \\
\text { de TI de negocio } \\
\text { para asegurar } \\
\text { sus logros futuros. }\end{array}$ & $\begin{array}{l}\text { - Garantizar la } \\
\text { pronta recuperación } \\
\text { de los servicios } \\
\text { (críticos) TI tras } \\
\text { un desastre. } \\
\text { - Establecer políticas } \\
\text { y procedimientos } \\
\text { que eviten, en la } \\
\text { medida de lo posible, } \\
\text { las perniciosas } \\
\text { consecuencias de } \\
\text { un desastre o causa } \\
\text { de fuerza mayor. }\end{array}$ \\
\hline
\end{tabular}

Fuente: (Cobit, 2015).

\section{RESUltados}

La presentación de los resultados de un trabajo de investigación debe responder a una serie de preguntas:
¿Qué se ha pretendido hacer?

El presente trabajo investigativo se ha pretendido demostrar los resultados que se pueden optimizar utilizando el sistema cobit para el control de las TI y SI.

¿Por qué razón es importante el tema?

La razón del tema propuesto es por la importancia que tiene en el área de auditoria informática o sistemas, ya que este abarca mucho las auditorias en organizaciones o empresas. ¿Qué se conoce ya sobre el tema?

El sistema Cobit en si es un modelo de control basado en el Gobierno TI para implementar sus estándares.

¿Cuál es la hipótesis del trabajo?

La hipótesis del trabajo es realizado para llegar a conocer la posibilidad o la incidencia que puede tener el sistema COBIT en la auditoria de los sistemas informáticos tanto en empresas públicas como privadas.

¿Cómo se ha llevado a cabo la investigación?

Para desarrollar este tema investigativo fue necesario investigar los conceptos claves de la utilización de sistemas COBIT y las metodologías que podemos incorporar en la Auditoria informática.

¿Cuáles son los resultados obtenidos?

En vista de algunos ejemplos que se citaron sobre la utilización del sistema COBIT en algunas empresas empleando el Gobierno TI, podemos verificar que la principal cualidad de este sistema es de la optimización de los recursos de Sistemas Informáticos.

¿Cómo se interpretan los resultados en relación a la hipótesis? Los resultados en relación a la hipótesis es la posibilidad de obtener mejores recursos en las TI

¿Cómo se relaciona la interpretación de los resultados con los conocimientos sobre el tema?

El conocimiento actual es la conceptualización de los sistemas COBIT y en vista de los resultados que se observan en algunos ejemplos es favorable la utilización del sistema para optimizar los recursos de TI y SI.

\section{REFERENCIAS BIBLIOGRÁFICAS}

Almanza Gomez, A. I. (2012). LA APLICACIÓN DE COBIT EN LAS ORGANIZACIONES ¿VALE LA PENA EL ESFUERZO? Universidad Militar Nueva Granada.

BITCompany (2015). Qué es CobiT: marco de referencia para la información y la tecnología - BITCompany via @bit_company.

Javeriana (2012). COBIT -Objetivos de Control para la Información y la Tecnología relacionada.

Reyes, V. (2015a). Estrategia de Gestión de Servicio de TI: 2.1 Definición y Antecedentes COBIT Y ITIL.

Reyes, V. (2015b). Estrategia de Gestión de Servicio de TI: 2.3. Fases de COBIT y ITIL.

Saffirio C., M. (2007). La COBIT y la Organización del Área Informática - Tecnologías de la Información y Procesos de Negocios (BPM).

Sperat, S. (2016). Estratega - Consultoría en estrategia y procesos - Todo lo que usted quería saber sobre Cobit 5 y no se animó a preguntar.

Zambrano, L. (2014). Antecedentes COBIT. 